\title{
A Potential Mechanism of Cardio-Renal Protection with Sodium-Glucose Cotransporter 2 Inhibitors: Amelioration of Renal Congestion
}

\author{
Satoru Kuriyama \\ Miho Clinic, and Jikei University School of Medicine, Tokyo, Japan
}

\section{Keywords}

Sodium-glucose cotransporter 2 inhibitors · Renal congestion · Renal protection · Diabetic kidney disease

\begin{abstract}
Background: This review considers anew the etiology of the cardio-renal protective effect of sodium-glucose cotransporter 2 (SGLT2) inhibitors by extending the discussion to renal congestion, inherent in diabetic kidney disease (DKD) even at an early stage of nephropathy in which heart failure (HF) or salt and water accumulation is asymptomatic. Summary: The interstitial fluid (IF) space of the kidney space plays a crucial role for tubulointerstitial inflammation, renal hypoxia, and ischemic injury, which often leads to renal progression. In DKD, as a result of hyperglycemic milieu, excessive salt and water can be accumulated in the IF space, creating renal congestion. I hypothesize that SGLT2 inhibitors cause a shift in extracellular water from the IF space to the intravascular space to compensate for the SGLT2 inhibitor-induced hypovolemia. This decrease in IF volume ameliorates the IF space milieu and may reduce inflammation, hypoxia, and ischemic injury. Message: The present review proposes a novel theory; unlike other hypoglycemic agents or diuretics, SGLT2 inhibitor could protect DKD from failing by improving latent renal congestion even without symptomatic HF.
\end{abstract}




\section{Kidney \\ Blood Pressure \\ Research}

\begin{tabular}{l|l}
\hline Kidney Blood Press Res 2019;44:449-456 \\
\hline DOI: 10.1159/000501081 & $\begin{array}{l}\text { @ 2019 The Author(s). Published by S. Karger AG, Basel } \\
\text { www.karger.com/kbr }\end{array}$ \\
\hline
\end{tabular}

Kuriyama: SGLT2 Inhibitors on Renal Congestion

\section{Introduction}

The EMPA-REG OUTCOME trial, the CANVAS Program, and the DECLAIRE-TIMI 58 have demonstrated that treatment with empagliflozin, canagliflozin, and dapagliflozin reduced hospitalization for heart failure (HF) and cardiovascular (CV) risks [1-3]. Both agents decreased albuminuria and retarded the progressive decline in renal function accompanied by an initial transient drop in the estimated glomerular filtration rate (eGFR) [4, 5]. They achieved composite outcome of a reduction in the eGFR and reduced the need for renalreplacement therapy and death from renal causes. The recently published CREDENCE trial recruiting patients with mild to moderate diabetic kidney disease (DKD) also confirmed that canagliflozin ameliorates renal prognosis even in individuals whose renal function decline in the neighboring of eGFR 30 to $<45 \mathrm{~mL} / \mathrm{min} / 1.73 \mathrm{~m}^{2}$ [6]. Influenced by these clinical trials, the effects of sodium-glucose cotransporter 2 (SGLT2) inhibitors on renal function have been widely studied in recent reviews [7-10]. Table 1 depicts the proposed cardio-renal protective mechanisms, most of them have obtained consensus. Of particular interest is that empagliflozin switches myocardial fuel utilization away from glucose toward ketone bodies, free fatty acid, and branched chain amino acid, thereby improving myocardial energetics, enhancing LV systolic function, and ameliorating adverse LV remodeling [11]. Despite their multifaceted discussion, there remain some unsolved mechanisms of renal benefits with SGLT2 inhibitors, which could better explain its cardio-renal protection.

\section{Evidence of Cardio-Renal Protection with SGLT2 Inhibitors}

There are many concomitant complications in diabetes mellitus (DM), including diabetic macro-, and micro-angiopathy of the kidney, hypertension, dyslipidemia, hyperuricemia, and obesity; all of these, if coexisting, accelerate decline in renal function. The pathophysiology of DKD has been explained by an increased activity of local renin-angiotensin-aldosterone system, sympathetic nerve hyperactivity, and the modulation of tubulo-glomerular feedback in an association with glomerular hyperfiltration $[12,13]$. For all events, the intrinsic characteristic in DKD is salt and water retention, an overhydrated condition. Indeed, excessive salt and water constantly creates a vicious cycle, worsening kidney function in a step-by-step manner $[14,15]$.

Congestion is a state of stagnant blood circulation in either capillaries or venous beds of the interstitial tissue. Severe to moderate congestion occurs as a clinical manifestation of circulatory failure, HF. Systemic congestive conditions such as severe edema, pulmonary, liver and renal congestion retain excessive $\mathrm{Na}$ and water in the interstitial fluid (IF) space, which eventually leads to a decline in renal function. Of note is that DKD is associated with a latent overhydrated state even long before progressing into end-stage renal failure and/or symptomatic HF.

In general, congestion can be treated with diuretics. Indeed, diuretic agents are, acutely or transiently, effective for any type of congestion; HF, pulmonary edema, liver congestion, abdominal congestion, and renal congestion. However, none of the diuretics are continually effective in improving a patient's prognosis. The diuretic effects of SGLT2 inhibitors seem unlikely to explain the benefits of this drug family because greater decreases in intravascular volume and net sodium balance are obtained by loop diuretics or thiazide, but these diuretic drugs do not reduce CV death and their effect of HF hospitalizations is much more modest [16]. Thus, the beneficial cardio-renal results observed in the aforementioned 4 large-scaled trials would suggest that SGLT2 inhibitors acted on the kidney differently from the conventional diuretics $[1-3,6]$. The renal protective effects elicited by SGLT2 inhibitors are originated from the dual excretory mechanisms of SGLT2 inhibitors: natriuresis and glycosuria. This unique pharmacological property may contribute to the improvement of renal interstitial congestion. 
Table 1. Cardio-renal protective mechanisms of SGLT2 inhibitors

$1 \quad$ Hemodynamic factors (natriuretic effects)

Reduction in BP

Modulation of TGF

Improvement of salt sensitivity

Improvement of sympathetic nerve hyperactivity

2 Metabolic factors (glycosuric effects)

Plasma glucose-lowering

Improvement of insulin sensitivity

Less glucose toxicity

Reduction in body weight

UA-lowering

TG and small dense LDL-C-lowering

3 Miscellaneous potential factors

Improvement of erythropoiesis and reduced oxidative stress

Inhibition of the NHE in heart and kidney

Restoration of energy efficiency in myocardial utilization by switching

Glucose to the more energy efficient metabolites, KB, FFA and BCAA

UA, uric acid; TG, triglyceride; LDL-C, LDL-cholesterol; NHE, Na-H exchanger; KB, ketone bodies; FFA, free fatty acid; BCAA, branched chain amino acid; BP, blood pressure; TGF, tubulo-glomerular feedback; SGLT2, sodium-glucose cotransporter 2 .

\section{Renal Interstitial Congestion and Renal Damage}

The structure of IF space has been clarified as a primary source of lymph and fluid compartment in the body. It is, basically, fluid-filled interstitial space and the presence of fluid has important implications for the physiological function of tissue as well as pathophysiological disorders such as hypertension and malignant tumors [17]. Increased Na concentration in the IF space of skin tissue, known as subcutaneous edema, has also become a center of attention because of its unique well-organized regulatory system [18]. A high concentration of salt is stored under the skin, stimulated by the lymphatic vascular endothelial growth factor mediated by macrophage mobilization. This system is regarded as a sort of buffering system of blood pressure that regulates the level of blood pressure at the time when excessive salt burden in hypervolemia and/or congestive HF becomes apparent.

Analogous to other organ tissues, the renal interstitial tissue and the IF space plays a crucial role not only being an anatomical supporting tissue of vasculature and tubules, but also being a responsible lesion for tubulointerstitial inflammation, renal hypoxia, and/or ischemic injury. Renal interstitial fibrosis and tubular atrophy, involved in renal anemia and inflammation, is a final common feature that contributes to the progression of CKD [19-21]. In addition to CKD, the role of glomerular hemodynamics and tubulointerstitial fibrosis is also very crucial in DKD [10, $12,13]$. These findings imply that perturbations to exacerbate interstitial milieu at the time of congestion or overhydration of IF space triggers the impairment of kidney function.

The pathophysiological importance of renal congestion and subsequent impaired renal function is a fairly new clinical concept [22]. Recent research clearly discloses that renal congestion is closely associated with decline in renal function. The reasons why congestion leads to renal damage are multifactorial and have not always been clearly understood. However, it is speculated that such mechanisms include attenuation of trans-glomerular pressure gradient, rise in renal interstitial pressure, local hypoxia, compression of the tubules, and increased luminal pressure, which are coexisting with the malfunction of tubulo-glomerular feedback, acti- 


\section{Kidney \\ Blood Pressure \\ Research}

\begin{tabular}{l|l}
\hline Kidney Blood Press Res 2019;44:449-456 \\
\hline DOI: 10.1159/000501081 & $\begin{array}{l}\text { @ 2019 The Author(s). Published by S. Karger AG, Basel } \\
\text { www.karger.com/kbr }\end{array}$ \\
\hline
\end{tabular}

Kuriyama: SGLT2 Inhibitors on Renal Congestion
Fig. 1. Renal congestion in diabetic kidney. Note that excessive overhydration and/or HF can result in a decline in renal function through renal congestion that could be brought about by hemodynamic, humoral and SN factors as well as other co-factors. CO, Cardiac output; HF, heart failure; RAAS, Renin angiotensin aldosterone system; SN, sympathetic nerve; GFR, glomerular filtration rate; IF, interstitial fluid; HT, hypertension; UA, uric acid; NSAID, nonsteroidal anti-inflammatory drug.

Fig. 2. Congestion of IF space in diabetic organs. In diabetic kidney, absolute or relative deficiency of insulin (a dotted arrow) inhibits glucose entering the ICF space. Under this condition, glucose in the IF space is prone to be elevated. SGLT2 inhibitors acts not only on renal tubules but on the IF space $\left({ }^{*}\right)$ to improve IF congestion by reducing the crystalloid osmotic pressure there, while the other diuretics act primarily only on the renal tubules. ICF, intracellular fluid; IF, interstitial fluid; IVF, intravascular fluid; Glu, glucose.
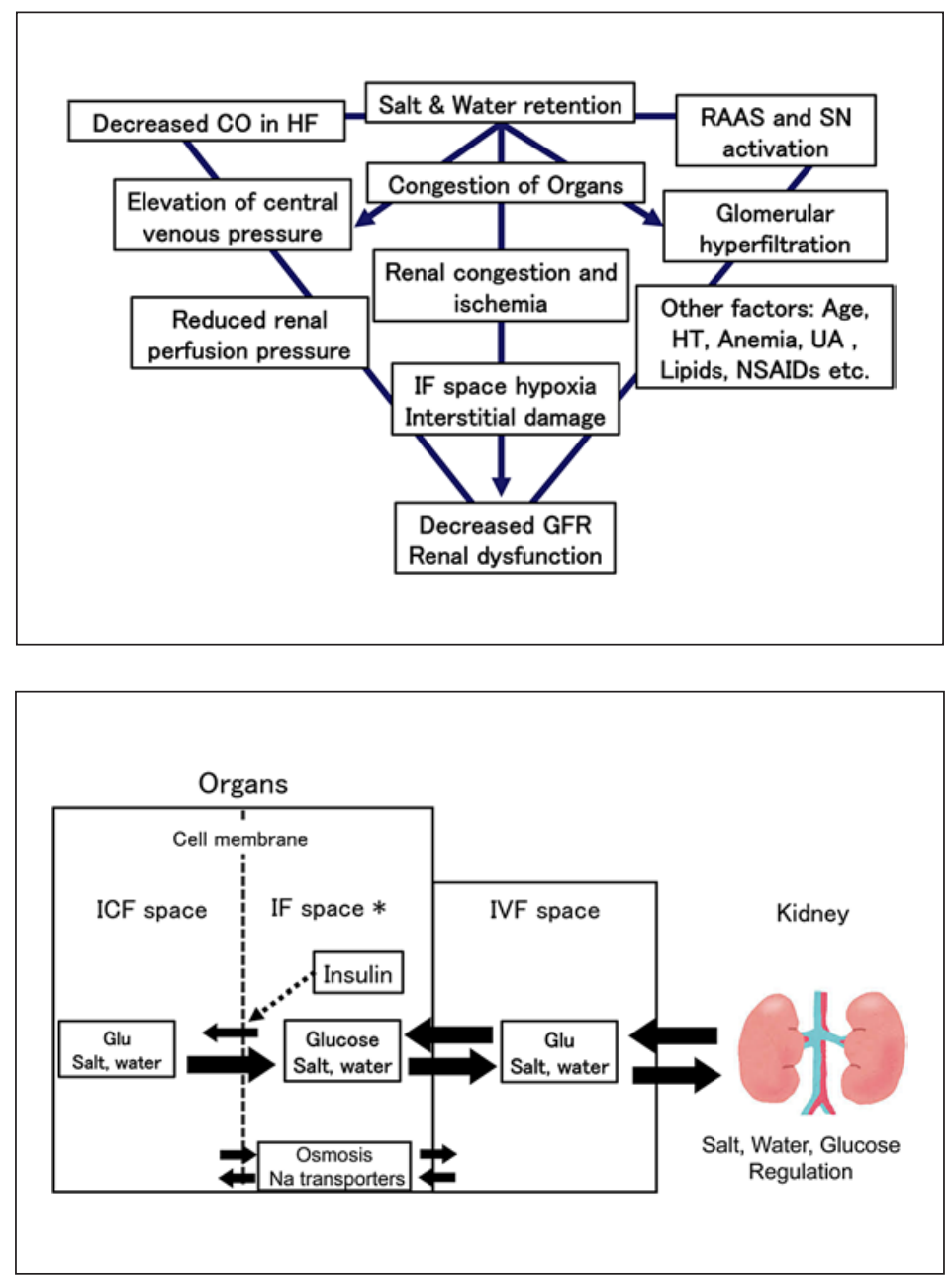

vation of the renin-angiotensin-aldosterone system, sympathetic nerve, and inflammatory mediators [23-26]. Increased central venous pressure and jugular venous pressure are also associated with impaired renal function $[27,28]$. The relationship between severe venous congestion and renal injury is complex [29]. The pathophysiology of renal injury in acute or chronic decompensated HF in DM is depicted in Figure 1 in which multiple factors are dependently and/or independently involved. It has been known that renal blood flow is preserved until cardiac index falls below $1.5 \mathrm{~L} / \mathrm{min} / \mathrm{m}^{2}$. In many patients, it is venous congestion rather than arterial underfilling that is associated with decreased renal blood flow and worsening of renal function [30].

The determinants of osmotic driving force across the different body spaces consist of the sum of serum $\mathrm{Na}$, plasma glucose and urea-nitrogen concentration. The crystalloid osmolarity pressure $(\mathrm{OP})$ is calculated; $\mathrm{OP}=2 \times($ serum $\mathrm{Na})+$ (plasma glucose $)+$ urea-nitrogen $/ 2.8$. In a diabetic state, either absolute or relative deficiency of insulin produces hyperglycemia, which increases OP in both IF and intravascular space. With an inability of glucose to appropriately enter intracellular space, increased OP in the IF space stimulates the accumulation of excessive salt and water in the IF space. Figure 2 simulates such a shifting of salt and water across the 3-compartments model, intracellular, IF and intravascular spaces in patients with DM. Of note is that increased renal venous pressure also exaggerates IF congestion, accumulating more salt and water in the IF space. The vicious cycle of increase in salt, water, and glucose in the IF space milieu is specific in diabetic nephropathy and concurrent diabetic HF. 


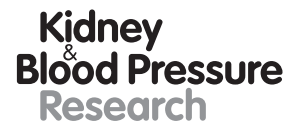

Kidney
Blood Pressure
Research \begin{tabular}{l|l}
\hline Kidney Blood Press Res 2019:44:449-456 \\
\hline DOI: 10.1159/000501081 & $\begin{array}{l}\text { @ 2019 The Author(s). Published by S. Karger AG, Basel } \\
\text { www.karger.com/kbr }\end{array}$ \\
\hline
\end{tabular}

Kuriyama: SGLT2 Inhibitors on Renal Congestion

Importantly, even without HF, DM has multiple innate high risk conditions that cause unfavorable physiological complications of the kidney.

From the diagnostic viewpoint, unlike pulmonary congestion and liver congestion that can be detected relatively easily by either ultrasonographical or radiographical examinations, there is no accurate method to assess renal congestion. A recent trial showed that the use of Doppler ultrasonography technique renders it capable of visualizing intrarenal hemodynamic changes [31]. There may be some additional surrogates to find renal congestion such as eGFR, BNP, NT-pro-BNP, and renal emerging marker such as kidney injury molecule 1, neutrophil gelatinase-associated lipocalin and/or cystatin C [26, 32]. However, unfortunately, none of them have been proven to be specific for renal congestion. In a daily clinical practice, it is difficult to evaluate renal congestion except for BW reduction or increase in hematocrit in response to SGLT2 inhibitor therapy.

\section{Effect of SGLT2 Inhibitors on Renal Interstitial Congestion}

The new therapeutic strategies for DKD have been updated and summarized in a recent review, in which SGLT2 inhibitors, GLP-1 analogs, DPP-4 inhibitors, thiazolidinedione, and mineralocorticoid antagonists are options for treating DKD [33]. Among these multiple candidates, SGLT2 inhibitors, supported firmly by the aforementioned 4 major large-scale clinical studies, are regarded as the most practical and promising cardio-renal protectors.

An appropriate removal of excessive salt and water by using drugs or other therapeutic intervention is crucial in HF. The use of various diuretics such as furosemide and bumetanide, and judicious use of ACE-I or ARB, mineralocorticoid antagonists, beta-blockers, sacubitril [34], and in the case of diuretic-resistance, extracorporeal ultrafiltration method and dialysis could be options. SGLT2 inhibitors may be on the list in the management of cardio-renal syndrome in the future because of their unique volume regulatory mechanism, which is basically different from that of the other diuretics. SGLT2 inhibition reduces both glucose and Na reabsorption in the proximal tubule. It also stimulates an osmotic diuresis effect in the distal tubule. With SGLT2 inhibitor, un-reabsorbed glucose flows into the distal nephron segment and as water is successively reabsorbed along the nephron, glucose concentration and tubular fluid osmolarity is increased, reducing the osmotic gradient between the tubular fluid and interstitial tissue. In this regard, the osmotic diuresis with SGLT2 inhibitors is basically different from that with other diuretics. Lee et al. [35] showed that approximately 15-35\% of total BW reduction is attributable to extracellular water (ECW) reduction. A part of this change may reflect the improvement of renal congestion in DKD. It should be emphasized that SGLT2 inhibitors antagonize Na-H exchanger of the kidney, enhancing natriuresis independently of the glycosuria effect; hence, the natriuretic effect will also be found in nondiabetic patients with lower glycosuria [36, 37].

Hallow et al. [38] hypothesized a differential regulatory mechanism of SGLT2 inhibitors. This idea is based on an assumption that osmotic diuresis induced by SGLT2 inhibitors is a distinctly different property from that of other diuretic classes. They compared blood and IF volume in response to 2 diuretic agents, dapagliflozin and bumetanide, and found that dapagliflozin produces twofold greater reduction in IF volume compared to blood volume, while the reduction in IF volume with bumetanide is only $78 \%$ of the reduction in blood volume, suggesting that SGLT2 inhibitors reduce IF volume to a greater extent than blood volume, and that SGLT2 inhibitors may provide better control of congestion without reducing effective circulation volume. This hypothetical idea was also in agreement with their clinical observations [39]. The specific pharmacological action of SGLT2 inhibitor's leads to greater electrolyte-free water clearance and results in greater fluid clearance from the IF space than from the intravascular space, potentially acting as a congestion reliever. It is also beneficial that 


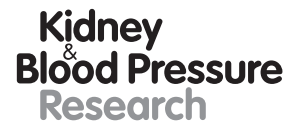

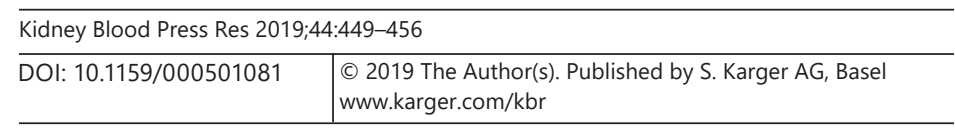

Kuriyama: SGLT2 Inhibitors on Renal Congestion

SGLT2 inhibitors have minimal impact on blood volume, arterial filling, and organ perfusion. There is no question that this difference in volume regulation by the clinical experiment using dapagliflozin and bumetanide in healthy subject can safely be extrapolated to DKD.

Of interest is that Hirose et al. [40] using body composition analyzer in diabetics, found that a daily dose of $20 \mathrm{mg}$ tofogliflozin for 8 weeks decreased total body water, ECW, and intracellular water and total body water, with an increase in hematocrit. This study strongly supports the idea that the SGLT2 inhibitor causes a shift in ECW from the IF space to the intravascular space to compensate for the SGLT2 inhibitor-induced hypovolemia and the alteration of the compartment ratio in ECW between IF space and intravascular space, again suggesting the benefit of SGLT2 inhibitors in reducing salt and water in the IF space. SGLT2 inhibitors lower osmotic pressure of the IF space, and at the same time removes glucose and salt through the kidney (Fig. 2). This sequential attenuating movement of Na and water in the IF space could help interstitial tissue recover from insulting factors such as inflammation, hypoxia and/or ischemia. Interestingly, an exploratory analysis from the EMPA-REG OUTCOME trial shows that changes in markers of plasma volume were the most important mediators of the reduction in risk of CV death with empagliflozin versus placebo [41]. Using indocyanine green indicator dilution method, Sha et al. [42] observed that canagliflozin provided a transient but substantial reduction in plasma volume. The decrease in plasma volume at week 1 was largely attenuated by week 12 despite a sustained increase in urinary glucose excretion over the 12 weeks of treatment, which can be explained by my hypothesis that SGLT2 inhibitors reduce IF volume.

From the therapeutic viewpoint, although experimental, Zhang et al. [43] has shown that luseogliflozin suppressed development of renal fibrosis and the prevention of peritubular capillary congestion/hemorrhage, increased vascular endothelial growth factor-A expression, and suppressed hypoxia in renal fibrosis animal model after renal ischemia/reperfusion injury. In addition, O'Neill et al. [44] demonstrated that SGLT2 inhibition with phlorizin in the streptozotocin-induced diabetic rats indeed mitigates interstitial hypoxia in the renal cortex. These basic studies are supportive of my current hypothesis that SGLT2 inhibitors serve as renal congestion remedies. All in all, it is conceivable that SGLT2 inhibitors improve glomerulopathy as well as tubulopathy and vasculopathy in DKD. In the not so distant future, treatment indication of SGLT2 inhibitors may expand not only to DKD but also to nondiabetic individuals with congestive disorders, CKD, and/or HF [45]. In fact, there is an ongoing clinical trial, the EMPA-TROPISM, studying the cardiac effects of empagliflozin for HF with reduced ejection fraction specifically in nondiabetic patients [46].

Taking all of the abovementioned evidence into consideration, a hypothesis of SGLT2 inhibitors being a renal congestion remedy appears to be very likely.

In summary, the present review will gain a new insight into the treatment of DM with SGLT2 inhibitors, in which renal congestion may be a risk for future progression. Unlike other hypoglycemic agents, the use of SGLT2 inhibitors for diabetic patients can be an appropriate way to provide a better milieu in the IF space of the kidney in DKD. However, solid clinical supporting studies must be performed in the near future.

\section{Conclusion}

SGLT2 inhibitors attenuate renal congestion by diminishing excessive salt and water from the IF space of the kidney, providing a better milieu for the renal interstitial tissue to recover. Thus, SGLT2 inhibitors can be a potential promising "Renal Congestion Remedy" to protect the kidney from failing in diabetics. 


\section{Statement of Ethics}

This article does not contain any studies performed with human participants or animals.

\section{Disclosure Statement}

No conflict of interest is disclosed.

\section{References}

1 Neal B, Perkovic V, Mahaffey KW, de Zeeuw D, Fulcher G, Erondu N, et al.; CANVAS Program Collaborative Group. Canagliflozin and Cardiovascular and Renal Events in Type 2 Diabetes. N Engl J Med. 2017 Aug;377(7): 644-57.

2 Zinman B, Wanner C, Lachin JM, Fitchett D, Bluhmki E, Hantel S, et al.; EMPA-REG OUTCOME Investigators. Empagliflozin, Cardiovascular Outcomes, and Mortality in Type 2 Diabetes. N Engl J Med. 2015 Nov;373(22): 2117-28.

3 Wiviott SD, Raz I, Bonaca MP, Mosenzon 0, Kato ET, Cahn A, et al.; DECLARE-TIMI 58 Investigators. Dapagliflozin and Cardiovascular Outcomes in Type 2 Diabetes. N Engl J Med. 2019 Jan;380(4):347-57.

4 Wanner C, Inzucchi SE, Lachin JM, Fitchett D, von Eynatten M, Mattheus M, et al.; EMPA-REG OUTCOME Investigators. Empagliflozin and Progression of Kidney Disease in Type 2 Diabetes. N Engl J Med. 2016 Jul;375(4): 323-34.

5 Heerspink HJ, Desai M, Jardine M, Balis D, Meininger G, Perkovic V. Canagliflozin Slows Progression of Renal Function Decline Independently of Glycemic Effects. J Am Soc Nephrol. 2017 Jan;28(1):368-75.

6 Perkovic V, Jardine MJ, Neal B, Bompoint S, Heerspink HJ, Charytan DM, et al.; CREDENCE Trial Investigators. Canagliflozin and Renal Outcomes in Type 2 Diabetes and Nephropathy. N Engl J Med. 2019 Jun 13;380(24): 2295-306.

7 Heerspink HJ, Kosiborod M, Inzucchi SE, Cherney DZ. Renoprotective effects of sodium-glucose cotransporter-2 inhibitors. Kidney Int. 2018 Jul; 94(1):26-39.

8 Georgianos PI, Divani M, Eleftheriadis T, Mertens PR, Liakopoulos V. SGLT-2 inhibitors in diabetic kidney disease: what lies behind their renoprotective properties? Curr Med Chem. 2018, Epub ahead of print.

9 Layton AT. Optimizing SGLT inhibitor treatment for diabetes with chronic kidney diseases. Biol Cybern. 2019 Apr;113(1-2):139-48.

10 de Albuquerque Rocha N, Neeland IJ, McCullough PA, Toto RD, McGuire DK. Effects of sodium glucose co-transporter 2 inhibitors on the kidney. Diab Vasc Dis Res. 2018 Sep;15(5):375-86.

11 Santos-Gallego CG, Requena-Ibanez JA, San Antonio R, Ishikawa K, Watanabe S, Picatoste B, et al. Empagliflozin Ameliorates Adverse Left Ventricular Remodeling in Nondiabetic Heart Failure by Enhancing Myocardial Energetics. J Am Coll Cardiol. 2019 Apr;73(15):1931-44.

12 Wanner C. EMPA-REG OUTCOME: The Nephrologist's Point of View. Am J Med. 2017 Jun;130(6 6S):S63-72.

13 Sano M. A new class of drugs for heart failure: SGLT2 inhibitors reduce sympathetic overactivity. J Cardiol. 2018 May; 71(5):471-6.

14 Sousa AG, Cabral JV, El-Feghaly WB, de Sousa LS, Nunes AB. Hyporeninemic hypoaldosteronism and diabetes mellitus: pathophysiology assumptions, clinical aspects and implications for management. World J Diabetes. 2016 Mar; 7(5):101-11.

15 Weir MR. Salt, hypertension, and proteinuria in diabetic nephropathy. Lancet Diabetes Endocrinol. 2014 May; 2(5):351-2.

16 Marx N, McGuire DK. Sodium-glucose cotransporter-2 inhibition for the reduction of cardiovascular events in high-risk patients with diabetes mellitus. Eur Heart J. 2016 Nov;37(42):3192-200.

17 Benias PC, Wells RG, Sackey-Aboagye B, Klavan H, Reidy J, Buonocore D, et al. Structure and Distribution of an Unrecognized Interstitium in Human Tissues. Sci Rep. 2018 Mar;8(1):4947.

18 Marvar PJ, Gordon FJ, Harrison DG. Blood pressure control: salt gets under your skin. Nat Med. 2009 May; 15(5):487-8.

19 Farris AB, Colvin RB. Renal interstitial fibrosis: mechanisms and evaluation. Curr Opin Nephrol Hypertens. 2012 May;21(3):289-300.

20 Nangaku M. Chronic hypoxia and tubulointerstitial injury: a final common pathway to end-stage renal failure. J Am Soc Nephrol. 2006 Jan;17(1):17-25.

21 Oliveira EA, Mak RH. Progression of chronic kidney disease in children - role of glomerular hemodynamics and interstitial fibrosis. Curr Opin Pediatr. 2018 Apr;30(2):220-7.

22 Afsar B, Ortiz A, Covic A, Solak Y, Goldsmith D, Kanbay M. Focus on renal congestion in heart failure. Clin Kidney J. 2016 Feb;9(1):39-47.

23 Blankstein R, Bakris GL. Renal hemodynamic changes in heart failure. Heart Fail Clin. 2008 Oct;4(4):411-23. 


\section{Kidney \\ Blood Pressure \\ Research}

\begin{tabular}{l|l}
\hline Kidney Blood Press Res 2019;44:449-456 \\
\hline DOI: 10.1159/000501081 & $\begin{array}{l}\text { @ } 2019 \text { The Author(s). Published by S. Karger AG, Basel } \\
\text { www.karger.com/kbr }\end{array}$ \\
\hline
\end{tabular}

24 Sambandam KK, Palmer BF. Effective use of loop diuretics in heart failure exacerbation: a nephrologist's view. Am J Med Sci. 2014 Feb;347(2):139-45.

25 Ruggenenti P, Remuzzi G. Worsening kidney function in decompensated heart failure: treat the heart, don't mind the kidney. Eur Heart J. 2011 Oct;32(20):2476-8.

26 Schrier RW. Role of diminished renal function in cardiovascular mortality: marker or pathogenetic factor? J Am Coll Cardiol. 2006 Jan;47(1):1-8.

27 Damman K, van Deursen VM, Navis G, Voors AA, van Veldhuisen DJ, Hillege HL. Increased central venous pressure is associated with impaired renal function and mortality in a broad spectrum of patients with cardiovascular disease. J Am Coll Cardiol. 2009 Feb;53(7):582-8.

28 Drazner MH, Rame JE, Stevenson LW, Dries DL. Prognostic importance of elevated jugular venous pressure and a third heart sound in patients with heart failure. N Engl J Med. 2001 Aug;345(8):574-81.

29 F Gnanaraj J, von Haehling S, Anker SD, Raj DS, Radhakrishnan J. The relevance of congestion in the cardiorenal syndrome. Kidney Int. 2013 Mar;83(3):384-91.

30 Ljungman S, Laragh JH, Cody RJ. Role of the kidney in congestive heart failure. Relationship of cardiac index to kidney function. Drugs. 1990;39 Suppl 4:10-21.

31 Iida N, Seo Y, Sai S, Machino-Ohtsuka T, Yamamoto M, Ishizu T, et al. Clinical implications of intrarenal hemodynamic evaluation by doppler ultrasonography in heart failure. JACC Heart Fail. 2016 Aug;4(8):674-82.

32 Pfister R, Müller-Ehmsen J, Hagemeister J, Hellmich M, Erdmann E, Schneider CA. NT-pro-BNP predicts worsening renal function in patients with chronic systolic heart failure. Intern Med J. 2011 Jun;41(6):467-72.

33 Lin YC, Chang YH, Yang SY, Wu KD, Chu TS. Update of pathophysiology and management of diabetic kidney disease. J Formos Med Assoc. 2018 Aug;117(8):662-75.

34 Ponikowski P, Voors AA, Anker SD, Bueno H, Cleland JG, Coats AJ, et al.; Authors/Task Force Members; Document Reviewers. 2016 ESC Guidelines for the diagnosis and treatment of acute and chronic heart failure: the Task Force for the diagnosis and treatment of acute and chronic heart failure of the European Society of Cardiology (ESC). Developed with the special contribution of the Heart Failure Association (HFA) of the ESC. Eur J Heart Fail. 2016 Aug;18(8):891-975.

35 Lee PC, Ganguly S, Goh SY. Weight loss associated with sodium-glucose cotransporter-2 inhibition: a review of evidence and underlying mechanisms. Obes Rev. 2018 Dec;19(12):1630-41.

36 Flores E, Santos-Gallego CG, Diaz-Mejía N, Badimon JJ. Do the SGLT-2 Inhibitors Offer More than Hypoglycemic Activity? Cardiovasc Drugs Ther. 2018 Apr;32(2):213-22.

37 Packer M, Anker SD, Butler J, Filippatos G, Zannad F. Effects of Sodium-Glucose Cotransporter 2 Inhibitors for the Treatment of Patients With Heart Failure: Proposal of a Novel Mechanism of Action. JAMA Cardiol. 2017 Sep;2(9):1025-9.

38 Hallow KM, Helmlinger G, Greasley PJ, McMurray JJ, Boulton DW. Why do SGLT2 inhibitors reduce heart failure hospitalization? A differential volume regulation hypothesis. Diabetes Obes Metab. 2018 Mar;20(3):479-87.

39 Hallow KM, Greasley PJ, Helmlinger G, Chu L, Heerspink HJ, Boulton DW. Evaluation of renal and cardiovascular protection mechanisms of SGLT2 inhibitors: model-based analysis of clinical data. Am J Physiol Renal Physiol. 2018 Nov;315(5):F1295-306.

40 Hirose S, Nakajima S, Iwahashi Y, Seo A, Takahashi T, Tamori Y. Impact of the 8-week administration of tofogliflozin for glycemic control and body composition in Japanese patients with type 2 diabetes mellitus. Intern Med. 2016;55(22):3239-45.

41 Inzucchi SE, Zinman B, Fitchett D, Wanner C, Ferrannini E, Schumacher M, et al. How Does Empagliflozin Reduce Cardiovascular Mortality? Insights From a Mediation Analysis of the EMPA-REG OUTCOME Trial. Diabetes Care. 2018 Feb;41(2):356-63.

42 Sha S, Polidori D, Heise T, Natarajan J, Farrell K, Wang SS, et al. Effect of the sodium glucose co-transporter 2 inhibitor canagliflozin on plasma volume in patients with type 2 diabetes mellitus. Diabetes Obes Metab. 2014 Nov; 16(11):1087-95.

43 Zhang Y, Nakano D, Guan Y, Hitomi H, Uemura A, Masaki T, et al. A sodium-glucose cotransporter 2 inhibitor attenuates renal capillary injury and fibrosis by a vascular endothelial growth factor-dependent pathway after renal injury in mice. Kidney Int. 2018 Sep;94(3):524-35.

44 O’Neill J, Fasching A, Pihl L, Patinha D, Franzén S, Palm F. Acute SGLT inhibition normalizes 02 tension in the renal cortex but causes hypoxia in the renal medulla in anaesthetized control and diabetic rats. Am J Physiol Renal Physiol. 2015 Aug;309(3):F227-34.

45 Wanner C, Marx N. SGLT2 inhibitors: the future for treatment of type 2 diabetes mellitus and other chronic diseases. Diabetologia. 2018 Oct;61(10):2134-9.

46 Santos-Gallego CG, Garcia-Ropero A, Mancini D, Pinney SP, Contreras JP, Fergus I, et al. Rationale and Design of the EMPA-TROPISM Trial (ATRU-4): Are the "Cardiac Benefits" of Empagliflozin Independent of its Hypoglycemic Activity? Cardiovasc Drugs Ther. 2019 Feb;33(1):87-95. 